\title{
LAT is essential for the mast cell stabilising effect of tHGA in IgE-mediated mast cell activation
}

\begin{abstract}
Mast cells play a central role in the pathogenesis of allergic reaction. Activation of mast cells by antigens is strictly dependent on the influx of extracellular calcium that involves a complex interaction between signalling molecules located within the cells. We have previously reported that tHGA, an active compound originally isolated from a local shrub known as Melicope ptelefolia, prevented IgE-mediated mast cell activation and passive systemic anaphylaxis by suppressing the release of interleukin-4 (IL-4) and tumour necrosis factor (TNF) $-\alpha$ from activated rat basophilic leukaemia (RBL)-2H3 cells. However, the mechanism of action (MOA) as well as the molecular target underlying the mast cell stabilising effect of tHGA has not been previously investigated. In this study, DNP-IgEsensitised RBL-2H3 cells were pre-treated with tHGA before challenged with DNP-BSA. To dissect the MOA of tHGA in IgE-mediated mast cell activation, the effect of tHGA on the transcription of IL-4 and TNF- $\alpha$ mRNA was determined using Real Time-Polymerase Chain Reaction (qPCR) followed by Calcium Influx Assay to confirm the involvement of calcium in the activation of mast cells. The protein lysates were analysed by using Western Blot to determine the effect of tHGA on various important signalling molecules in the LAT-PLC $\gamma$ MAPK and PI3K-NFאB pathways. In order to identify the molecular target of tHGA in IgEmediated mast cell activation, the LAT and LAT2 genes in RBL-2H3 cells were knockeddown by using RNA interference to establish a LAT/LAT2 competition model. The results showed that tHGA inhibited the transcription of IL-4 and TNF- $\alpha$ as a result of the suppression of calcium influx in activated RBL-2H3 cells. The results from Western Blot revealed that tHGA primarily inhibited the LAT-PLC $\gamma$-MAPK pathway with partial inhibition on the PI3K-p65 pathway without affecting Syk. The results from RNAi further demonstrated that tHGA failed to inhibit the release of mediators associated with mast cell degranulation under the LAT/LAT2 competition model in the absence of LAT. Collectively, this study concluded that the molecular target of tHGA could be LAT and may provide a basis for the development of a mast cell stabiliser which targets LAT.
\end{abstract}

Keyword: tHGA; RBL-2H3; IgE-mediated mast cell activation; LAT; LAT 2; Melicope ptelefolia 